VOLUME 27 (2020) 127-146

DOI: $10.24330 /$ ieja.662996

\title{
CLASSIFICATION OF THREE-DIMENSIONAL ZEROPOTENT ALGEBRAS
}

\author{
Anton Cedilnik and Marjan Jerman \\ Received: 15 February 2019; Revised: 23 October 2019; Accepted: 29 October 2019 \\ Communicated by Abdullah Harmancı
}

\begin{abstract}
The classification of three-dimensional zeropotent algebras over an arbitrary field is given. It is complete up to the individual properties of the ground field.
\end{abstract}

Mathematics Subject Classification (2010): 17A30, 17A60

Keywords: Zeropotent algebra, anti-commutative algebra, non-associative algebra, Lie algebra

\section{Introduction}

A non-associative algebra $\mathcal{A}$ over an arbitrary field $\mathbb{F}$ is zeropotent if $x^{2}=0$ for all $x \in \mathcal{A}$. If $\operatorname{chr} \mathbb{F}$, the characteristic of the field $\mathbb{F}$, is different from two, we usually use the term anti-commutative algebra.

There are four non-isomorphic types of zeropotent algebras of dimension less than three. All of them are Lie algebras. In the two-dimensional case there exists a basis $\{a, b\} \subset \mathcal{A}$ with

$$
a b=\sigma a, \sigma \in\{0,1\} .
$$

The classification of three-dimensional zeropotent algebras over an algebraically closed field $\mathbb{F}$ with characteristic different from two was determined in [2] and of those over the field of real numbers in [4]. In the thesis [1], there is a classification of three-dimensional Lie algebras.

We shall generalize these results to algebras over an arbitrary field up to the solvability of certain equations and conditions of quadratic character in this field.

For $x, y, z \in \mathcal{A}$ let

$$
J(x, y, z)=(x y) z+(y z) x+(z x) y
$$

denote the Jacobian. A zeropotent algebra is Lie if $J(x, y, z)=0$ for all $x, y, z \in \mathcal{A}$, and it is Maltsev if $J(x, y, x z)=J(x, y, z) x$ for all $x, y, z \in \mathcal{A}$. An algebra is binaryLie if any two of its elements generate a Lie subalgebra. Any Lie algebra is Maltsev and any Maltsev algebra is binary-Lie. 


\section{Preliminaries}

Proposition 2.1 (Kuzmin, [3]). A three-dimensional binary-Lie algebra is Lie.

Proof. There is nothing to prove if there exist two elements which generate the algebra.

Now suppose that any two linearly independent elements generate a two-dimensional subalgebra. Considering $(*)$ choose a basis $\{a, b, c\}$ with

$$
a b=\sigma a, a c=\alpha a+\beta c, b c=\gamma b+\delta c .
$$

The examination of the products $a(b-c), b(c-a)$ and $c(a-b)$ gives $\beta=0$, $\delta=-\sigma$ and $\gamma=\alpha$, respectively. Since the Jacobian of any three linearly dependent elements in a zeropotent algebra is always 0 , it is enough to check that $J(a, b, c)=$ 0 .

A minor generalization of this proof would show that each binary-Lie algebra with the property that any two linearly independent elements generate a twodimensional subalgebra is Lie.

Throughout the paper we shall use the following notation:

Given three vectors

$$
x=\left(x_{1}, x_{2}, x_{3}\right), y=\left(y_{1}, y_{2}, y_{3}\right), z=\left(z_{1}, z_{2}, z_{3}\right) \in \mathbb{F}^{3},
$$

define

$$
D=\operatorname{det}(x, y, z)
$$

and its $2 \times 2$ cofactors

$$
\begin{aligned}
& A=y_{2} z_{3}-y_{3} z_{2}, \quad B=y_{3} z_{1}-y_{1} z_{3}, \quad C=y_{1} z_{2}-y_{2} z_{1}, \\
& K=x_{3} z_{2}-x_{2} z_{3}, \quad L=x_{1} z_{3}-x_{3} z_{1}, \quad M=x_{2} z_{1}-x_{1} z_{2}, \\
& U=x_{2} y_{3}-x_{3} y_{2}, \quad V=x_{3} y_{1}-x_{1} y_{3}, \quad W=x_{1} y_{2}-x_{2} y_{1} .
\end{aligned}
$$

The identities in the following lemma will be heavily used in solving large systems of equations in the rest of the paper.

Lemma 2.2. The following relations hold:

$$
\begin{gathered}
y_{1} A+y_{2} B+y_{3} C=z_{1} A+z_{2} B+z_{3} C=0, \\
x_{1} K+x_{2} L+x_{3} M=z_{1} K+z_{2} L+z_{3} M=0, \\
x_{1} U+x_{2} V+x_{3} W=y_{1} U+y_{2} V+y_{3} W=0 ; \\
D=x_{1} A+x_{2} B+x_{3} C=y_{1} K+y_{2} L+y_{3} M=z_{1} U+z_{2} V+z_{3} W ;
\end{gathered}
$$




$$
\begin{aligned}
& L W-M V=x_{1} D, \quad M U-K W=x_{2} D, \quad K V-L U=x_{3} D, \\
& C V-B W=y_{1} D, \quad A W-C U=y_{2} D, \quad B U-A V=y_{3} D, \\
& B M-C L=z_{1} D, \quad C K-A M=z_{2} D, \quad A L-B K=z_{3} D .
\end{aligned}
$$

\section{The three classes}

We shall divide all three-dimensional zeropotent algebras into three classes, according to the properties of their subalgebras of dimension two:

(1) The first class: This class contains all algebras which contain at least one two-dimensional subalgebra with zero multiplication.

(2) The second class: This class contains all algebras which contain two-dimensional subalgebras, none of which has zero multiplication.

(3) The third class: This class contains algebras without two-dimensional subalgebras.

Note that any two algebras from different classes are non-isomorphic.

In the first class the vector space basis of an algebra $\mathcal{A}$ will consist of two linearly independent elements from a two-dimensional subalgebra with zero multiplication, and an additional element from its complement. In the second class the basis of $\mathcal{A}$ will consist of two linearly independent elements $a$ and $b$ from a two-dimensional subalgebra with $a b=a$ (considering $(*)$ ), and an additional element from its complement. The basis of the algebras from the third class will consist of any two linearly independent elements together with their product.

The multiplication table of a zeropotent algebra $\mathcal{A}$ is thus given by Table 1 with $(\sigma, \tau) \in\{(0,0),(1,0),(0,1)\}$ and $\pi_{i}, \rho_{i} \in \mathbb{F}$ for $i=1,2,3$.

\begin{tabular}{|c|ccc|}
\hline$\cdot$ & $a$ & $b$ & $c$ \\
\hline$a$ & 0 & $\sigma a+\tau c$ & $\pi_{1} a+\pi_{2} b+\pi_{3} c$ \\
$b$ & $-\sigma a-\tau c$ & 0 & $\rho_{1} a+\rho_{2} b+\rho_{3} c$ \\
$c$ & $-\pi_{1} a-\pi_{2} b-\pi_{3} c$ & $-\rho_{1} a-\rho_{2} b-\rho_{3} c$ & 0 \\
\hline
\end{tabular}

TABLE 1 . The multiplication table of a zeropotent algebra $\mathcal{A}$.

For $f, g \in \mathcal{A}$ let

$$
f=x_{1} a+x_{2} b+x_{3} c, g=y_{1} a+y_{2} b+y_{3} c \text { for some } x_{i}, y_{i} \in \mathbb{F} .
$$

Note that $f$ and $g$ are linearly dependent if and only if $U=V=W=0$. 
Then,

$$
f g=\left(\rho_{1} U-\pi_{1} V+\sigma W\right) a+\left(\rho_{2} U-\pi_{2} V\right) b+\left(\rho_{3} U-\pi_{3} V+\tau W\right) c .
$$

The linear independence of the elements $f, g$ and $f g$ can be examined by calculating the determinant $\operatorname{det}(f, g, f g)$ of the corresponding coefficients relative to the basis $\{a, b, c\}$. It is equal to $E(U, V, W)$ where $E$ is the homogeneous polynomial

$$
E(x, y, z)=x^{2} \rho_{1}-y^{2} \pi_{2}+z^{2} \tau-y z \pi_{3}+x z\left(\rho_{3}+\sigma\right)+x y\left(\rho_{2}-\pi_{1}\right) .
$$

According to Table 1, the three classes of algebras can be alternatively described by:

(1) The first class: $(\sigma, \tau)=(0,0)$.

(2) The second class: $(\sigma, \tau)=(1,0)$ and $\pi_{2} \rho_{3} \neq \pi_{3} \rho_{2}$.

(3) The third class: $(\sigma, \tau)=(0,1)$ and $E(x, y, z) \neq 0$ if $(x, y, z) \neq(0,0,0)$.

In the second class the product of any two linearly independent elements is not zero, therefore the solution of the non-homogeneous system

$$
\rho_{1} U-\pi_{1} V+W=\rho_{2} U-\pi_{2} V=\rho_{3} U-\pi_{3} V=0
$$

is trivial and the determinant of the last homogeneous subsystem $\pi_{2} \rho_{3}-\pi_{3} \rho_{2}$ is non-zero.

In the third class the condition $\operatorname{det}(f, g, f g) \neq 0$ for all linearly independent $f$ and $g$ implies that the underlying field $\mathbb{F}$ cannot be algebraically closed or even quasialgebraically closed. Also, by Chevalley-Warning's theorem, the field $\mathbb{F}$ cannot be finite.

Note that $U, V$ and $W$ can take arbitrary values. A triple $(U=0, V, W)$ can be obtained by choosing $x_{1}=1, x_{2}=x_{3}=y_{1}=0, y_{2}=W, y_{3}=-V$; and a triple $(U \neq 0, V, W)$ is for example a result of $x_{1}=-V, x_{2}=y_{2}=U, x_{3}=0$, $y_{1}=-V-W U^{-1}, y_{3}=1$.

To use less space we shall write Table 1 in a more compact form as

$$
\text { Algebra : } \begin{array}{|rr|lll|lll|}
\sigma & \tau & \pi_{1} & \pi_{2} & \pi_{3} & \rho_{1} & \rho_{2} & \rho_{3} \\
\hline
\end{array}
$$

Now suppose that the $\Phi: \mathcal{A} \rightarrow \mathcal{A}^{\prime}$ is an isomorphism of two three-dimensional zeropotent algebras with bases $\{a, b, c\}$ and $\left\{a^{\prime}, b^{\prime}, c^{\prime}\right\}$ and that

$\hat{a}=\Phi(a)=x_{1} a^{\prime}+x_{2} b^{\prime}+x_{3} c^{\prime}, \hat{b}=\Phi(b)=y_{1} a^{\prime}+y_{2} b^{\prime}+y_{3} c^{\prime}, \hat{c}=\Phi(c)=z_{1} a^{\prime}+z_{2} b^{\prime}+z_{3} c^{\prime}$.

Then, $\{\hat{a}, \hat{b}, \hat{c}\}$ is also a basis of $\mathcal{A}^{\prime}$ and

$$
D=\operatorname{det}(\hat{a}, \hat{b}, \hat{c}) \neq 0 \text {. }
$$


By $\hat{\sigma}, \hat{\tau}, \hat{\pi}_{1}, \hat{\pi}_{2}, \hat{\pi}_{3}, \hat{\rho}_{1}, \hat{\rho}_{2}$ and $\hat{\rho}_{3}$ denote the structure constants corresponding to the multiplication in the basis $\left\{a^{\prime}, b^{\prime}, c^{\prime}\right\}$.

Two algebras from different classes are not isomorphic, therefore

$$
\hat{\sigma}=\sigma \text { and } \hat{\tau}=\tau .
$$

Since $\Phi(a b)=\Phi(a) \Phi(b)=\hat{a} \hat{b}$

$$
\begin{aligned}
U \rho_{1}-V \pi_{1}+W \sigma & =x_{1} \sigma+z_{1} \tau, \\
U \rho_{2}-V \pi_{2} & =x_{2} \sigma+z_{2} \tau, \\
U \rho_{3}-V \pi_{3}+W \tau & =x_{3} \sigma+z_{3} \tau .
\end{aligned}
$$

The multiplication table shows that $\hat{a} \hat{b}=\hat{\sigma} \hat{a}+\hat{\tau} \hat{b}=\sigma \hat{a}+\tau \hat{b}$, therefore

$$
\begin{gathered}
A U \rho_{1}-B V \pi_{2}+C W \tau-C V \pi_{3}+C U \rho_{3}+A W \sigma+B U \rho_{2}-A V \pi_{1}=D \sigma, \\
K U \rho_{1}-L V \pi_{2}+M V \tau-M V \pi_{3}+M U \rho_{3}+K W \sigma+L U \rho_{2}-K V \pi_{1}=0, \\
U^{2} \rho_{1}-V^{2} \pi_{2}+W^{2} \tau-V W \pi_{3}+U W\left(\rho_{3}+\sigma\right)+U V\left(\rho_{2}-\pi_{1}\right)=D \tau .
\end{gathered}
$$

In the same way the coordinates of $\hat{a} \hat{c}$ and $\hat{b} \hat{c}$ show that

$$
\begin{gathered}
\hat{\pi}_{1}=-D^{-1}\left(A K \rho_{1}-B L \pi_{2}+C M \tau-C L \pi_{3}+C K \rho_{3}+A M \sigma+B K \rho_{2}-A L \pi_{1}\right), \\
\hat{\pi}_{2}=-D^{-1}\left(K^{2} \rho_{1}-L^{2} \pi_{2}+M^{2} \tau-L M \pi_{3}+K M\left(\rho_{3}+\sigma\right)+K L\left(\rho_{2}-\pi_{1}\right)\right), \\
\hat{\pi}_{3}=x_{1} \pi_{3}+x_{2}\left(\rho_{3}-\sigma\right)-x_{3}\left(\pi_{1}+\rho_{2}\right) ; \\
\hat{\rho}_{1}=D^{-1}\left(A^{2} \rho_{1}-B^{2} \pi_{2}+C^{2} \tau-B C \pi_{3}+A C\left(\rho_{3}+\sigma\right)+A B\left(\rho_{2}-\pi_{1}\right),\right. \\
\hat{\rho}_{2}=D^{-1}\left(A K \rho_{1}-B L \pi_{2}+C M \tau-B M \pi_{3}+A M \rho_{3}+C K \sigma+A L \rho_{2}-B K \pi_{1}\right), \\
\hat{\rho}_{3}=y_{1} \pi_{3}+y_{2}\left(\rho_{3}-\sigma\right)-y_{3}\left(\pi_{1}+\rho_{2}\right)+\sigma .
\end{gathered}
$$

We shall often use some additional equations which will make our calculations easier:

$$
\begin{gathered}
\hat{\pi}_{1} x_{1}+\hat{\pi}_{2} y_{1}=x_{1} z_{3} \pi_{1}-x_{1} z_{1} \pi_{3}-K \rho_{1}+x_{3} z_{1} \rho_{2}-x_{2} z_{1} \rho_{3}+x_{1} z_{2} \sigma, \\
\hat{\pi}_{1} x_{2}+\hat{\pi}_{2} y_{2}=x_{3} z_{2} \pi_{1}+L \pi_{2}-x_{1} z_{2} \pi_{3}+x_{2} z_{3} \rho_{2}-x_{2} z_{2} \rho_{3}+x_{2} z_{2} \sigma, \\
\hat{\pi}_{1} x_{3}+\hat{\pi}_{2} y_{3}=x_{3} z_{3} \pi_{1}-x_{3} z_{1} \pi_{3}+x_{3} z_{3} \rho_{2}-x_{3} z_{2} \rho_{3}+x_{2} z_{3} \sigma-M \tau ; \\
\hat{\rho}_{1} x_{1}+\hat{\rho}_{2} y_{1}=y_{1} z_{3} \pi_{1}-y_{1} z_{1} \pi_{3}+A \rho_{1}+y_{3} z_{1} \rho_{2}-y_{2} z_{1} \rho_{3}+\left(y_{1} z_{2}-z_{1}\right) \sigma, \\
\hat{\rho}_{1} x_{2}+\hat{\rho}_{2} y_{2}=y_{3} z_{2} \pi_{1}-B \pi_{2}-y_{1} z_{2} \pi_{3}+y_{2} z_{3} \rho_{2}-y_{2} z_{2} \rho_{3}+\left(y_{2} z_{2}-z_{2}\right) \sigma, \\
\hat{\rho}_{1} x_{3}+\hat{\rho}_{2} y_{3}=y_{3} z_{3} \pi_{1}-y_{3} z_{1} \pi_{3}+y_{3} z_{3} \rho_{2}-y_{3} z_{2} \rho_{3}+\left(y_{2}-1\right) z_{3} \sigma+C \tau ; \\
\hat{\pi}_{1}+\hat{\rho}_{2}=z_{3}\left(\pi_{1}+\rho_{2}\right)-z_{1} \pi_{3}-z_{2}\left(\rho_{3}-\sigma\right) .
\end{gathered}
$$


In the rest of this section we shall simplify the above equations by using special properties of each class.

3.1. The first class $(\sigma, \tau)=(0,0)$. If $\pi_{3} \neq 0$, the change of basis

$$
\hat{a}=\rho_{3} a-\pi_{3} b, \hat{b}=a, \hat{c}=c,
$$

results in $\hat{\pi}_{3}=0$. Therefore, we can assume that $\pi_{3}=0$ is an invariant and that the multiplication is given by Table 2 .

\begin{tabular}{|c|ccc|}
\hline$\cdot$ & $a$ & $b$ & $c$ \\
\hline$a$ & 0 & 0 & $\pi_{1} a+\pi_{2} b$ \\
$b$ & 0 & 0 & $\rho_{1} a+\rho_{2} b+\rho_{3} c$ \\
$c$ & $-\pi_{1} a-\pi_{2} b$ & $-\rho_{1} a-\rho_{2} b-\rho_{3} c$ & 0 \\
\hline
\end{tabular}

TABLE 2. The first class: $(\sigma, \tau)=(0,0)$.

The system of equations for the first class is reduced to

$$
\begin{gathered}
D=z_{1} U+z_{2} V+z_{3} W \neq 0, \\
U \rho_{1}-V \pi_{1}=U \rho_{2}-V \pi_{2}=U \rho_{3}=0, \\
x_{2} \rho_{3}=x_{3}\left(\pi_{1}+\rho_{2}\right) ; \\
\hat{\pi}_{1} x_{1}+\hat{\pi}_{2} y_{1}=\left(x_{1} z_{3}-x_{3} z_{1}\right) \pi_{1}+\left(x_{2} z_{3}-x_{3} z_{2}\right) \rho_{1}, \\
\hat{\pi}_{1} x_{2}+\hat{\pi}_{2} y_{2}=\left(x_{1} z_{3}-x_{3} z_{1}\right) \pi_{2}+\left(x_{2} z_{3}-x_{3} z_{2}\right) \rho_{2}, \\
\hat{\pi}_{1} x_{3}+\hat{\pi}_{2} y_{3}=\left(x_{2} z_{3}-x_{3} z_{2}\right) \rho_{3}, \\
\hat{\rho}_{1} x_{1}+\hat{\rho}_{2} y_{1}=y_{1} z_{3} \pi_{1}+\left(y_{2} z_{3}-y_{3} z_{2}\right) \rho_{1}+y_{3} z_{1} \rho_{2}-y_{2} z_{1} \rho_{3}, \\
\hat{\rho}_{1} x_{2}+\hat{\rho}_{2} y_{2}=y_{3} z_{2} \pi_{1}+\left(y_{1} z_{3}-y_{3} z_{1}\right) \pi_{2}+y_{2} z_{3} \rho_{2}-y_{2} z_{2} \rho_{3}, \\
\hat{\rho}_{1} x_{3}=\hat{\pi}_{1} y_{3}, \\
\hat{\rho}_{3}=y_{2} \rho_{3}-y_{3}\left(\pi_{1}+\rho_{2}\right), \\
\hat{\rho}_{1}=D^{-1}\left(A^{2} \rho_{1}-B^{2} \pi_{2}+A C \rho_{3}+A B\left(\rho_{2}-\pi_{1}\right)\right), \\
\hat{\rho}_{2}=D^{-1}\left(A K \rho_{1}-B L \pi_{2}+A M \rho_{3}+A L \rho_{2}-B K \pi_{1}\right), \\
\hat{\pi}_{1}+\hat{\rho}_{2}=z_{3}\left(\pi_{1}+\rho_{2}\right)-z_{2} \rho_{3} .
\end{gathered}
$$


3.2. The second class $(\sigma, \tau)=(1,0)$. The transformation

$$
\hat{a}=a, \hat{b}=b, \hat{c}=-\pi_{1} b+c,
$$

gives $\hat{\pi}_{1}=0$. Therefore, we can assume that $\pi_{1}=0$. The multiplication in an algebra from the second class is given by Table 3 .

\begin{tabular}{|c|ccc|}
\hline$\cdot$ & $a$ & $b$ & $c$ \\
\hline$a$ & 0 & $a$ & $\pi_{2} b+\pi_{3} c$ \\
$b$ & $-a$ & 0 & $\rho_{1} a+\rho_{2} b+\rho_{3} c$ \\
$c$ & $-\pi_{2} b-\pi_{3} c$ & $-\rho_{1} a-\rho_{2} b-\rho_{3} c$ & 0 \\
\hline
\end{tabular}

TABLE 3 . The second case: $(\sigma, \tau)=(1,0), \pi_{2} \rho_{3} \neq \pi_{3} \rho_{2}$.

The system of equations for the second class is reduced to:

$$
\begin{gathered}
D=z_{1} U+z_{2} V+z_{3} W \neq 0 \\
U \rho_{1}+W=x_{1}, U \rho_{2}-V \pi_{2}=x_{2}, U \rho_{3}-V \pi_{3}=x_{3} \\
\hat{\pi}_{2} y_{1}=\left(x_{2} z_{3}-x_{3} z_{2}\right) \rho_{1}+\left(x_{3} z_{1}-x_{1} z_{3}\right) \rho_{2}+\left(x_{2} z_{1}-z_{2} x_{1}\right) \rho_{3}+\hat{\rho}_{2} x_{1}, \\
\hat{\pi}_{2} y_{2}=\left(x_{1} z_{3}-x_{3} z_{1}\right) \pi_{2}+\left(x_{2} z_{1}-x_{1} z_{2}\right) \pi_{3}+\hat{\rho}_{2} x_{2}, \\
\hat{\pi}_{2} y_{3}=x_{2} z_{3}-x_{3} z_{2}+\hat{\rho}_{2} x_{3}, \\
\hat{\pi}_{3}=x_{1} \pi_{3}+x_{2}\left(\rho_{3}-1\right)-x_{3} \rho_{2} ; \\
\hat{\rho}_{1} x_{1}=\left(y_{2} z_{3}-y_{3} z_{2}\right) \rho_{1}+\left(y_{3} z_{1}-y_{1} z_{3}\right) \rho_{2}+\left(y_{1} z_{2}-y_{2} z_{1}\right) \rho_{3}-z_{1}, \\
\hat{\rho}_{1} x_{2}=\left(y_{1} z_{3}-y_{3} z_{1}\right) \pi_{2}+\left(y_{2} z_{1}-y_{1} z_{2}\right) \pi_{3}-z_{2}, \\
\hat{\rho}_{1} x_{3}=y_{2} z_{3}-y_{3} z_{2}-z_{3}, \\
\hat{\rho}_{2}=-\left(z_{1} \pi_{3}+z_{2}\left(\rho_{3}-1\right)-z_{3} \rho_{2}\right), \\
\hat{\rho}_{3}=1+y_{1} \pi_{3}+y_{2}\left(\rho_{3}-1\right)-y_{3} \rho_{2} .
\end{gathered}
$$

3.3. The third class $(\sigma, \tau)=(0,1)$. The multiplication table for an algebra in this class will be even simpler. First suppose that $\pi_{3} \neq 0$. In the new basis

$$
\hat{a}=\rho_{3} a-\pi_{3} b, \hat{b}=a, \hat{c}=\pi_{3} c,
$$

we still have $\hat{c}=\hat{a} \hat{b}$ and $\operatorname{det}(\hat{a}, \hat{b}, \hat{c})=\pi_{3}^{2} \neq 0$. The product $\hat{a} \hat{c}$ is a linear combination of $\hat{a}$ and $\hat{b}$, hence we may assume in advance that $\pi_{3}=0$. 
Now suppose that $\rho_{3} \neq 0$. Let us show that the transformation

$$
\hat{a}=a, \hat{b}=\left(\pi_{1}+\rho_{2}\right) b+\rho_{3} c, \hat{c}=\pi_{1} \rho_{3} a+\pi_{2} \rho_{3} b+\left(\pi_{1}+\rho_{2}\right) c,
$$

defines a new basis such that $\hat{c}=\hat{a} \hat{b}$.

If $\pi_{2}$ were a square in $\mathbb{F}, E\left(0,1, \sqrt{\pi_{2}}\right)$ would equal 0 which contradicts the condition that $E(x, y, z) \neq 0$ if $(x, y, z) \neq(0,0,0)$. Therefore,

$$
\operatorname{det}(\hat{a}, \hat{b}, \hat{c})=\left(\pi_{1}+\rho_{2}\right)^{2}-\pi_{2} \rho_{3}^{2} \neq 0 .
$$

Because of

$$
\operatorname{det}(\hat{a}, \hat{b}, \hat{a} \hat{c})=\operatorname{det}(\hat{a}, \hat{b}, \hat{b} \hat{c})=0,
$$

we can further assume that $\rho_{3}=0$.

The third transformation

$$
\hat{a}=a, \hat{b}=\pi_{1} a+\pi_{2} b, \hat{c}=\pi_{2} c,
$$

is valid since $\operatorname{det}(\hat{a}, \hat{b}, \hat{c})=\pi_{2}^{2}$ and $E(0,1,0)=-\pi_{2} \neq 0$. This time $\hat{a} \hat{c}=\pi_{2} \hat{b}$, therefore we can also assume that $\pi_{1}=0$.

Finally, we may assume that $\rho_{2} \in\{0,1\}$ : in the case $\rho_{2} \neq 0$ we can apply the transformation

$$
\hat{a}=\rho_{2}^{-1} a, \hat{b}=b, \hat{c}=\rho_{2}^{-1} c,
$$

after which $\hat{\rho}_{2}=1$.

In this class the multiplication is given by Table 4 with $\lambda=-\pi_{2}, \mu=\rho_{1}$ and $\omega=\rho_{2}$.

\begin{tabular}{|c|ccc|}
\hline$\cdot$ & $a$ & $b$ & $c$ \\
\hline$a$ & 0 & $c$ & $-\lambda b$ \\
$b$ & $-c$ & 0 & $\mu a+\omega b$ \\
$c$ & $\lambda b$ & $-\mu a-\omega b$ & 0 \\
\hline
\end{tabular}

TABLE 4. The third case: $(\sigma, \tau)=(0,1), \omega \in\{0,1\}, x^{2} \mu+y^{2} \lambda+$ $z^{2}+x y \omega \neq 0$ if $(x, y, z) \neq(0,0,0)$.

The system of equations for the third class is as follows:

$$
\begin{gathered}
D=z_{1} U+z_{2} V+z_{3} W \neq 0, \\
U \mu=z_{1}, V \lambda=z_{2}, W=z_{3}, \\
x_{3} \omega=y_{3} \omega=\left(z_{3}-1\right) \omega=0, \\
A K \mu+B L \lambda+C M+x_{2} y_{1} \omega=0 ;
\end{gathered}
$$




$$
\begin{gathered}
\hat{\lambda} y_{1}=\left(x_{3} z_{2}-x_{2} z_{3}\right) \mu, \\
\hat{\lambda} y_{2}=\left(x_{1} z_{3}-x_{3} z_{1}\right) \lambda-x_{2} \omega, \\
\hat{\lambda} y_{3}=x_{2} z_{1}-x_{1} z_{2}, \\
\hat{\mu} x_{1}=\left(y_{2} z_{3}-y_{3} z_{2}\right) \mu-y_{1} \omega, \\
\hat{\mu} x_{2}=\left(y_{3} z_{1}-y_{1} z_{3}\right) \lambda, \\
\hat{\mu} x_{3}=y_{1} z_{2}-y_{2} z_{1} .
\end{gathered}
$$

The equation $J(a, b, c)=0$ determines which three-dimensional zeropotent algebras are Lie:

Proposition 3.1. An algebra with the multiplication given by Table 1 is a Lie algebra if and only if

$$
\pi_{3} \rho_{1}-\pi_{1} \rho_{3}=\rho_{2} \sigma, \pi_{2} \rho_{3}-\pi_{3} \rho_{2}=\pi_{2} \sigma \text { and } \pi_{3} \sigma=\left(\pi_{1}+\rho_{2}\right) \tau .
$$

An algebra with the multiplication from Table 2 is Lie if and only if

$$
\pi_{1} \rho_{3}=\pi_{2} \rho_{3}=0
$$

An algebra with the multiplication from Table 3 is Lie if and only if

$$
\pi_{2} \neq 0, \pi_{3}=\rho_{2}=0 \text { and } \rho_{3}=1 .
$$

An algebra with the multiplication from Table 4 is Lie if and only if

$$
\omega=0 .
$$

Remark 3.2. It is not difficult to prove that all algebras from the classes $(1,0)$ and $(0,1)$ are simple, having no non-trivial ideals.

\section{Classification within the first class $(0,0)$}

The dimension of the subalgebra $\operatorname{lin}(\mathcal{A A})$, the linear span of the set of all products $a b, a, b \in \mathcal{A}$, is either 0,1 or 2 . These dimensions are invariant under isomorphisms.

The case $\operatorname{dim} \operatorname{lin}(\mathcal{A A})=0$ corresponds to the zero algebra:

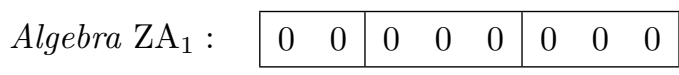

Now let us examine the second possibility, $\operatorname{dim} \operatorname{lin}(\mathcal{A A})=1$.

The products $a c$ and $b c$ in Table 2 are linearly dependent, i.e.,

$$
\pi_{1} \rho_{2}-\pi_{2} \rho_{1}=\pi_{1} \rho_{3}=\pi_{2} \rho_{3}=0
$$


If $a c \neq 0$, then $\rho_{3}=0$. The change of basis

$$
\hat{a}=x a+b, \hat{b}=a, \hat{c}=c \quad \text { with } x \pi_{1}+\rho_{1}=x \pi_{2}+\rho_{2}=0,
$$

results in $\hat{a} \hat{c}=0$. Hence, we may assume that $\pi_{1}=\pi_{2}=0$ and that $b c \neq 0$.

Now suppose that $\rho_{3} \neq 0$. The transformation

$$
\hat{a}=a, \hat{b}=\rho_{2} b+\rho_{3} c, \hat{c}=b
$$

gives $\hat{\rho}_{3}=0$, therefore, $\rho_{3}=0$ can be taken as a new invariant.

From the system of equations for the first class we can see that any choice of $\rho_{2} \neq 0$ implies $\hat{\rho}_{2} \neq 0$. The transformation

$$
\hat{a}=a, \hat{b}=\rho_{1} a+\rho_{2} b, \hat{c}=\rho_{2}^{-1} c
$$

fixes $\hat{\rho}_{2}=1$ and $\hat{\rho}_{1}=0$.

For a reason which will be clarified later, we change the basis once more to

$$
\hat{a}=b, \hat{b}=b-a, \hat{c}=c .
$$

Thus we get the algebra

$$
\text { Algebra } \mathrm{ZA}_{2}(0): \begin{array}{|ll|lll|lll|}
0 & 0 & 1 & 0 & 0 & 1 & 0 & 0 \\
\hline
\end{array}
$$

The reason for the additional zero in $\mathrm{ZA}_{2}(0)$ will also be explained later.

In the remaining case when $\rho_{2}=0$, the transformation

$$
\hat{a}=a, \hat{b}=\rho_{1}^{-1} b, \hat{c}=c,
$$

yields new invariants $\hat{\rho}_{1}=1$ and $\hat{\rho}_{2}=0$. The corresponding algebra is

$$
\text { Algebra } \mathrm{ZA}_{3} \text { : } \quad 0 \begin{array}{|ll|lll|lll|}
0 & 0 & 0 & 0 & 0 & 1 & 0 & 0 \\
\hline
\end{array}
$$

Now let $\operatorname{dim} \operatorname{lin}(\mathcal{A A})=2$. The products $a c$ and $b c$ from Table 2 are linearly independent. The conditions

$$
U \rho_{1}-V \pi_{1}=U \rho_{2}-V \pi_{2}=U \rho_{3}=0
$$

from the system of equations for the first class are equivalent to $U b c=V a c$, which implies $U=V=0$ and $x_{3}=y_{3}=0$. Note that $W \neq 0$ since $D=z_{1} U+z_{2} V+z_{3} W \neq$ 0 .

If $\rho_{3} \neq 0$, then $x_{2}=0$ and $x_{1} y_{2} z_{3} \neq 0$. The transformation

$$
\hat{a}=a, \hat{b}=\rho_{3}^{-1} b, \hat{c}=c,
$$

gives $\hat{\rho}_{3}=1$. Thus, we can choose a new invariant $\rho_{3}=1$.

If $\pi_{2} \neq 0$, we can use the following formulation:

$$
\hat{a}=a, \hat{b}=\pi_{1} \pi_{2}^{-1} a+b, \hat{c}=\pi_{2}^{-1}\left(\pi_{1}^{2} \pi_{2}^{-1}+\rho_{1}\right) a+\pi_{2}^{-1}\left(\pi_{1}+\rho_{2}\right) b+\pi_{2}^{-1} c,
$$


while if $\pi_{2}=0$, we can define

$$
\hat{a}=a, \hat{b}=b, \hat{c}=\pi_{1}^{-1} \rho_{1} a+\pi_{1}^{-1} \rho_{2} b+\pi_{1}^{-1} c,
$$

thus obtaining the following algebras:

$$
\begin{array}{l|rl|lll|lll|}
\text { Algebra } Z_{4}: & 0 & 0 & 0 & 1 & 0 & 0 & 0 & 1 \\
\text { Algebra } Z_{5}: & 0 & 0 & 1 & 0 & 0 & 0 & 0 & 1 \\
\hline
\end{array}
$$

From now on we can suppose that $\rho_{3}=0$, which implies $\hat{\rho}_{3}=0$ and $\pi_{1} \rho_{2} \neq \pi_{2} \rho_{1}$. The system of equations determining the first class is thus significantly simplified:

$$
\begin{gathered}
z_{3}\left(x_{1} y_{2}-x_{2} y_{1}\right) \neq 0 ; \\
\hat{\pi}_{1}=z_{3}\left(x_{1} y_{2}-x_{2} y_{1}\right)^{-1}\left(y_{2}\left(x_{1} \pi_{1}+x_{2} \rho_{1}\right)-y_{1}\left(x_{1} \pi_{2}+x_{2} \rho_{2}\right)\right), \\
\hat{\pi}_{2}=z_{3}\left(x_{1} y_{2}-x_{2} y_{1}\right)^{-1}\left(x_{1}\left(x_{1} \pi_{2}+x_{2} \rho_{2}\right)-x_{2}\left(x_{1} \pi_{1}+x_{2} \rho_{1}\right)\right), \\
\hat{\rho}_{1}=z_{3}\left(x_{1} y_{2}-x_{2} y_{1}\right)^{-1}\left(y_{2}\left(y_{1} \pi_{1}+y_{2} \rho_{1}\right)-y_{1}\left(y_{1} \pi_{2}+y_{2} \rho_{2}\right)\right), \\
\hat{\rho}_{2}=z_{3}\left(x_{1} y_{2}-x_{2} y_{1}\right)^{-1}\left(x_{1}\left(y_{1} \pi_{2}+y_{2} \rho_{2}\right)-x_{2}\left(y_{1} \pi_{1}+y_{2} \rho_{1}\right)\right) .
\end{gathered}
$$

Now suppose that $\rho_{2} \neq 0$ and that no change of the basis results in $\hat{\rho}_{2}=0$. Then, for any choice of $W \neq 0$ the following system

$$
x_{1} y_{2}-x_{2} y_{1}=W, x_{1}\left(y_{1} \pi_{2}+y_{2} \rho_{2}\right)-x_{2}\left(y_{1} \pi_{1}+y_{2} \rho_{1}\right)=0
$$

is not solvable. This happens precisely when it has a zero determinant

$$
y_{1}^{2} \pi_{2}+y_{1} y_{2}\left(\rho_{2}-\pi_{1}\right)-y_{2}^{2} \rho_{1}=0
$$

for all $y_{1}, y_{2} \in \mathbb{F}$. Therefore, $\pi_{2}=\rho_{1}=\rho_{2}-\pi_{1}=0$. After the transformation

$$
\hat{a}=a, \hat{b}=b, \hat{c}=\pi_{1}^{-1} c
$$

we get the algebra

$$
\text { Algebra } \mathrm{ZA}_{6}: \quad \begin{array}{|ll|lll|lll|}
0 & 0 & 1 & 0 & 0 & 0 & 1 & 0 \\
\hline
\end{array}
$$

On the other hand, if there is a basis in which $\rho_{2}=0$, we can take this $\rho_{2}$ as a new invariant. In the basis

$$
\hat{a}=\rho_{1} a, \hat{b}=b, \hat{c}=c,
$$

$\hat{\rho}_{1}=1$ and $\rho_{1}=1$ can be also taken as an invariant. Since $\hat{\pi}_{1}=z_{3} \pi_{1}$, the cases $\pi_{1} \neq 0$ and $\pi_{1}=0$ define non-isomorphic algebras.

If $\pi_{1} \neq 0$, we have $\hat{\pi}_{1}=1$ in the basis

$$
\hat{a}=a, \hat{b}=b, \hat{c}=\pi_{1}^{-1} c,
$$


and we can take another invariant $\pi_{1}=1$. The simplified system of equations for the first class shows that the algebras with different $\pi_{2}$ are non-isomorphic. Alternatively, we could use [2, Proposition 2.1] to show that for the matrices

$$
A_{1}=\left[\begin{array}{ccc}
1 & 0 & 0 \\
-1 & -\lambda_{1} & 0 \\
0 & 0 & 0
\end{array}\right] \text { and } A_{2}=\left[\begin{array}{ccc}
1 & 0 & 0 \\
-1 & -\lambda_{2} & 0 \\
0 & 0 & 0
\end{array}\right]
$$

there exists an invertible matrix $X$ such that $A_{2} \operatorname{det}(X)=X^{\top} A_{1} X$ if and only if $\lambda_{1}=\lambda_{2}$.

In this way we obtain a family of algebras which we denote as

$$
\text { Algebra } \mathrm{ZA}_{2}(\lambda \neq 0): \quad \begin{array}{|ll|lll|lll|}
0 & 0 & 1 & \lambda & 0 & 1 & 0 & 0 \\
\hline
\end{array}
$$

since for $\lambda=0$ the above formulation would give us the algebra which was previously denoted as $\mathrm{ZA}_{2}(0)$.

In the case when $\pi_{1}=0$, the simplified system of equations implies that $\hat{\pi}_{2}=$ $z_{3}^{2} \pi_{2}$ and the result depends on the quadratic character of $\pi_{2}$. There exist two alternatives, i.e.

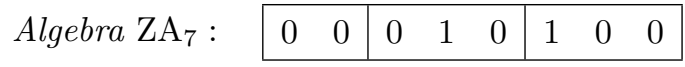

and

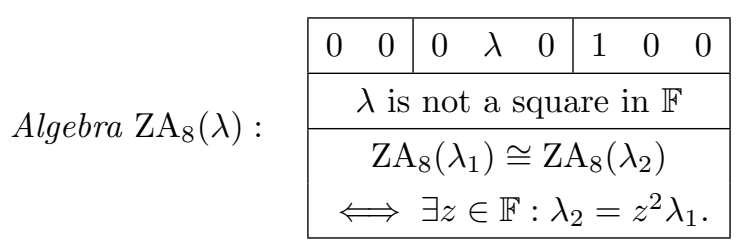

Again, the isomorphic pairs could be determined using [2, Proposition 2.1].

\section{Classification within the second class $(1,0)$}

Firstly, we shall classify Lie algebras; these are the algebras with

$$
\pi_{2} \neq 0, \pi_{3}=\rho_{2}=0, \rho_{3}=1 \text {. }
$$

The transformation

$$
\hat{a}=a, \hat{b}=b, \hat{c}=\pi_{2}^{-1} c
$$

results in $\hat{\pi}_{2}=1$ and we can take $\pi_{2}=1$ as an invariant.

We shall rename the only remaining parameter $\rho_{1}$ by $\lambda$.

If there is a basis in which $\lambda=0$, we get the algebra

$$
\text { Algebra } \mathrm{ZA}_{9}: \quad \begin{array}{|ll|lll|lll|}
\hline 1 & 0 & 0 & 1 & 0 & 0 & 0 & 1 \\
\hline
\end{array}
$$


In the remaining cases $\lambda \neq 0$ and no transformation yields $\hat{\lambda}=0$. Since the choice of basis

$$
\hat{a}=a, \hat{b}=b, \hat{c}=\frac{\lambda}{2} a+c \text { if } \operatorname{chr} \mathbb{F} \neq 2
$$

results in $\hat{\lambda}=0$, we conclude that $\operatorname{chr} \mathbb{F}=2$. Another transformation

$$
\hat{a}=a, \hat{b}=y a+b, \hat{c}=y b+c, y \in \mathbb{F}
$$

shows that $\lambda$ cannot be a square, therefore the field $\mathbb{F}$ is not finite. After some routine work with the system of equations for the second class we get the following description

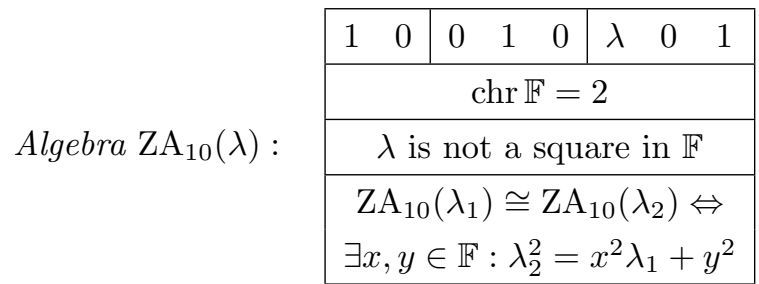

From now on we can suppose that the algebras are strictly non-Lie, i.e., at least one of the conditions

$$
\pi_{2} \neq 0, \pi_{3}=\rho_{2}=0, \rho_{3}=1
$$

does not hold.

Before continuing our classification we shall investigate how many two-dimensional subalgebras exist in such an algebra. Suppose that the pair

$$
p=x_{1} a+x_{2} b+x_{3} c, q=y_{1} a+y_{2} b+y_{3} c
$$

forms a basis of a subalgebra. At least one of the determinants $U, V$ or $W$ must be non-zero. A necessary and sufficient condition for the $\operatorname{span} \operatorname{lin}\{p, q\}$ to be a subalgebra is that

$$
\operatorname{det}(p, q, p q)=0=E(U, V, W)=U^{2} \rho_{1}-V^{2} \pi_{2}-V W \pi_{3}+U W\left(\rho_{3}+1\right)+U V \rho_{2} .
$$

The $\operatorname{span} \operatorname{lin}\{a, b\}$ is a subalgebra if $x_{3}=y_{3}=0$. If this is not the case, we may assume that

$$
\begin{gathered}
p=x_{1} a+x_{2} b, q=y_{1} a+y_{2} b+c ; \\
U=x_{2}, V=-x_{1}, W=x_{1} y_{2}-x_{2} y_{1} ; x_{1} \neq 0 \text { or } x_{2} \neq 0 .
\end{gathered}
$$

If $x_{2}=0$, we may require that $x_{1}=1$ and $y_{1}=0$. With the new notation $z=y_{2}$ we get

$$
p=a, q=z b+c, \operatorname{det}(p, q, p q)=-\pi_{2}+z \pi_{3} .
$$


If $x_{2} \neq 0$, we may take $x_{2}=1$ and $y_{2}=0$. In the new notation $y=y_{1}$ and $x=x_{1}$,

$$
\begin{gathered}
p=x a+b, q=y a+c ; \\
\operatorname{det}(p, q, p q)=\rho_{1}-x^{2} \pi_{2}-x y \pi_{3}-y\left(\rho_{3}+1\right)-x \rho_{2} .
\end{gathered}
$$

Therefore, if there is another two-dimensional subalgebra besides $\operatorname{lin}\{a, b\}$, at least one of the following equations is solvable:

$$
z \pi_{3}=\pi_{2},\left(\rho_{3}+1+x \pi_{3}\right) y=\rho_{1}-x \rho_{2}-x^{2} \pi_{2} .
$$

Since the existence of subalgebras is invariant under isomorphisms, we may follow the classification by determining non-Lie algebras with only one two-dimensional subalgebra. For such algebras the following conditions must hold:

(a) at least one of the conditions $\pi_{2} \neq 0, \pi_{3}=\rho_{2}=0$ or $\rho_{3}=1$ is not true;

(b) $\pi_{3}=0 \neq \pi_{2}$;

(c) $\rho_{3}+1+x \pi_{3}=0 \neq \rho_{1}-x \rho_{2}-x^{2} \pi_{2}$ for all $x \in \mathbb{F}$.

These conditions imply that $\pi_{3}=0$ and that $\rho_{3}=-1$. We shall take $\pi_{3}$ and $\rho_{3}$ for new invariants.

Firstly, suppose that $\operatorname{chr} \mathbb{F}=2$. By (a), $\rho_{2} \neq 0$ and the transformation

$$
\hat{a}=\pi_{2}^{-1} \rho_{2} a, \hat{b}=b, \hat{c}=\rho_{2}^{-1} c
$$

yields new invariants $\hat{\pi}_{2}=\hat{\rho}_{2}=1$. Solving the system of equations for the second class gives

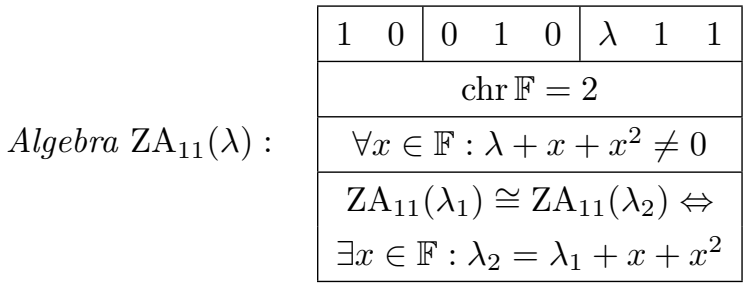

It remains to examine the case $\operatorname{chr} \mathbb{F} \neq 2$. The change of basis

$$
\hat{a}=\pi_{2}^{-1} a, \hat{b}=-\frac{1}{2} \pi_{2}^{-1} \rho_{2} a+b, \hat{c}=-\frac{1}{2} \rho_{2} b+c,
$$

results in $\hat{\pi}_{2}=1$ and $\hat{\rho}_{2}=0$, hence we may assume that $\pi_{2}=1$ and that $\rho_{2}=0$. A straightforward calculations yield the algebras

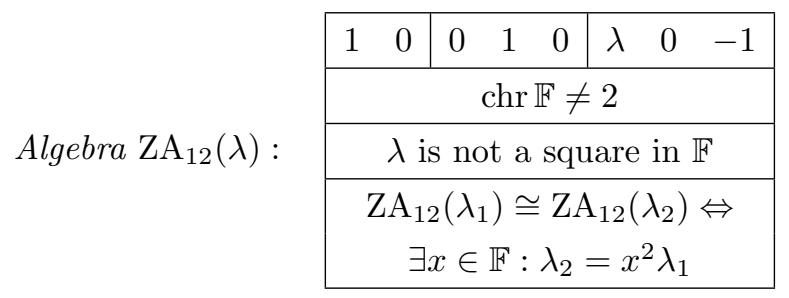


From now on we shall investigate non-Lie algebras with more than one subalgebra. In this setting:

(d) at least one of the conditions $\pi_{2} \neq 0, \pi_{3}=\rho_{2}=0$ or $\rho_{3}=1$ is false;

(e) at least one of the equations

$$
z \pi_{3}=\pi_{2},\left(\rho_{3}+1+x \pi_{3}\right) y=\rho_{1}-x \rho_{2}-x^{2} \pi_{2}
$$

is solvable.

Suppose first that $\pi_{2} \neq 0$ and that $\hat{\pi}_{2} \neq 0$ regardless of the choice of a basis. The transformation

$$
\hat{a}=a, \hat{b}=b, \hat{c}=\pi_{2} \pi_{3}{ }^{-1} a+\pi_{2} b+\pi_{3} c
$$

gives $\hat{\pi}_{2}=0$, hence $\pi_{3}=0$ (and $\pi_{2} \rho_{3} \neq 0$ ) is true in an arbitrary basis. Another transformation

$$
\hat{a}=\rho_{1} a+\left(\rho_{3}+1\right) c, \hat{b}=\rho_{2}\left(\pi_{2} \rho_{3}\right)^{-1} a-\rho_{3}^{-1} b, \hat{c}=\rho_{1}\left(\rho_{3}+1\right)^{-1} \rho_{3} a+\rho_{2} b+\rho_{3} c
$$

yields $\hat{\pi}_{2}=0$. Since

$$
\operatorname{det}(\hat{a}, \hat{b}, \hat{c})=\rho_{2}^{2}\left(\rho_{3}+1\right)\left(\pi_{2} \rho_{3}\right)^{-1},
$$

this implies that $\rho_{2}\left(\rho_{3}+1\right)=0$ and $\hat{\rho}_{2}\left(\hat{\rho}_{3}+1\right)=0$ for all changes of the basis.

The following transformation

$$
\hat{a}=a, \hat{b}=a+b, \hat{c}=\pi_{2} b+c
$$

makes $\hat{\rho}_{2}\left(\hat{\rho}_{3}+1\right)=\pi_{2}\left(1-\rho_{3}^{2}\right)$. Thus, $\rho_{3}^{2}=1$. By $(\mathrm{d}), \rho_{2}=0$ implies $\rho_{3} \neq 1$, therefore $\rho_{3}=-1$ and $\operatorname{chr} \mathbb{F} \neq 2$. The conclusion $\rho_{3}=-1$ also follows from $\rho_{2} \neq 0$.

Thus, we got $\pi_{3}=0$ and $\rho_{3}=-1$, together with the following two demands:

(f) at least one of the conditions $\rho_{2}=0$ or $\rho_{3}=1$ is false;

(g) the equation $\rho_{1}-x \rho_{2}-x^{2} \pi_{2}=0$ is solvable, i.e., there is a $\varphi \in \mathbb{F}$ such that

$$
\rho_{1}-\varphi \rho_{2}-\varphi^{2} \pi_{2}=0 .
$$

If $\operatorname{chr} \mathbb{F}=2$, the condition (f) implies that $\rho_{2} \neq 0$. In this case the transformation

$$
\hat{a}=\left(\rho_{2}+\varphi \pi_{2}\right) b+c, \hat{b}=\varphi a+b, \hat{c}=a
$$

results in $\hat{\pi}_{3}=\rho_{2} \neq 0$, which is not true. Hence chr $\mathbb{F} \neq 2$. The transformation

$$
\begin{array}{r}
\hat{a}=\left(\rho_{2}+\varphi \pi_{2}\right) b-c, \hat{b}=\varphi a+b, \\
\hat{c}=-\pi_{2}^{-1}\left(\pi_{2} \rho_{2} \varphi+2 \pi_{2} \rho_{1}+\rho_{2}^{2}\right) a+\pi_{2} \varphi b+c
\end{array}
$$

defines a new basis if $\operatorname{det}(\hat{a}, \hat{b}, \hat{c})=-\pi_{2}^{-1}\left(4 \pi_{2} \rho_{1}+\rho_{2}^{2}\right) \neq 0$. In this basis $\hat{\pi}_{2}=0$, therefore $4 \pi_{2} \rho_{1}+\rho_{2}^{2}=0$. 
A straightforward calculation using the system of equations for the second class shows that no other transformation of the basis gives $\hat{\pi}_{2}=0$.

To further simplify the multiplication table, use

$$
\hat{a}=a, \hat{b}=-\rho_{2}\left(2 \pi_{2}\right)^{-1} a+b, \hat{c}=-\rho_{2}\left(2 \pi_{2}\right)^{-1} b+\pi_{2}^{-1} c
$$

to get $\hat{\pi}_{2}=1$ and $\hat{\rho}_{1}=\hat{\rho}_{2}=0$ and the new algebra

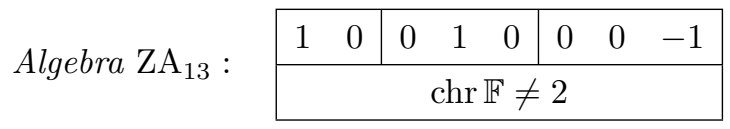

It remains to investigate the case of non-Lie algebras with more than one subalgebra and with $\pi_{2}=0$ in a suitable basis. In this case $\pi_{2}$ will be considered an invariant. Conditions (d) and (e) are automatically fulfilled. Also, we should not forget that $0=\pi_{2} \rho_{3} \neq \pi_{3} \rho_{2}$.

The transformation

$$
\hat{a}=\pi_{3}^{-1} a, \hat{b}=\rho_{1} \rho_{2}^{-1} a+b, \hat{c}=\rho_{2}^{-1} c
$$

yields another three invariants $\pi_{3}=\rho_{2}=1$ and $\rho_{1}=0$. We shall rename the only remaining parameter $\rho_{3}$ as $\lambda$.

The system of equations for the second class shows that the multiplication table is preserved by the following transformation:

$$
\begin{gathered}
\hat{a}=\left(1+x_{2}+x_{3}-x_{2} \lambda\right) a+x_{2} b+x_{3} c, \\
\hat{b}=\left(x_{3}-x_{2} \lambda\right) a+\left(1+x_{2}+x_{3}\right) b+\left(x_{3} \lambda-x_{2}\right) c, \\
\hat{c}=x_{2} a-x_{3} b+\left(1+x_{2}+x_{3}-x_{3} \lambda\right) c, \\
\text { with } x_{2}^{2}+x_{3}-x_{2} x_{3} \lambda+x_{2} x_{3}+x_{3}^{2}+x_{2}=0 .
\end{gathered}
$$

Any such transformation yields $\hat{\lambda}=\lambda$, hence all these algebras are non-isomorphic. Thus we obtained

$$
\text { Algebra } \mathrm{ZA}_{14}(\lambda): \begin{array}{|ll|lll|lll|}
\hline 1 & 0 & 0 & 0 & 1 & 0 & 1 & \lambda \\
\hline
\end{array}
$$




\section{Classification within the third class $(0,1)$}

Firstly suppose that we are dealing with the non-Lie case $\omega=1$. A simple calculation using the system of equations for the third class yields the algebras

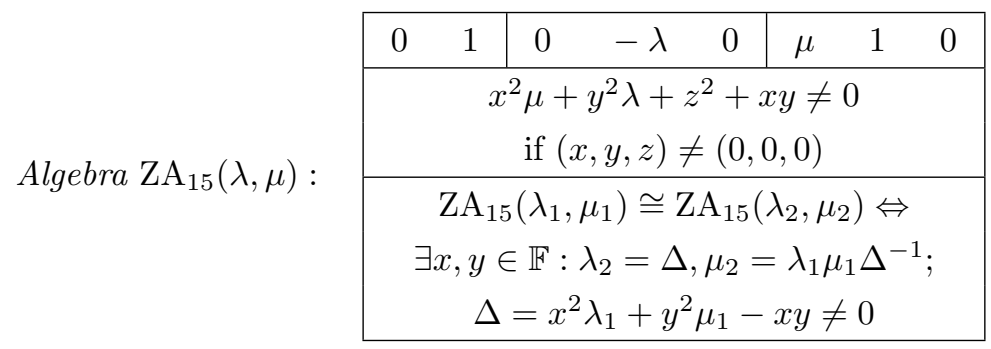

In the Lie case with $\omega=0$, we obtain the algebras

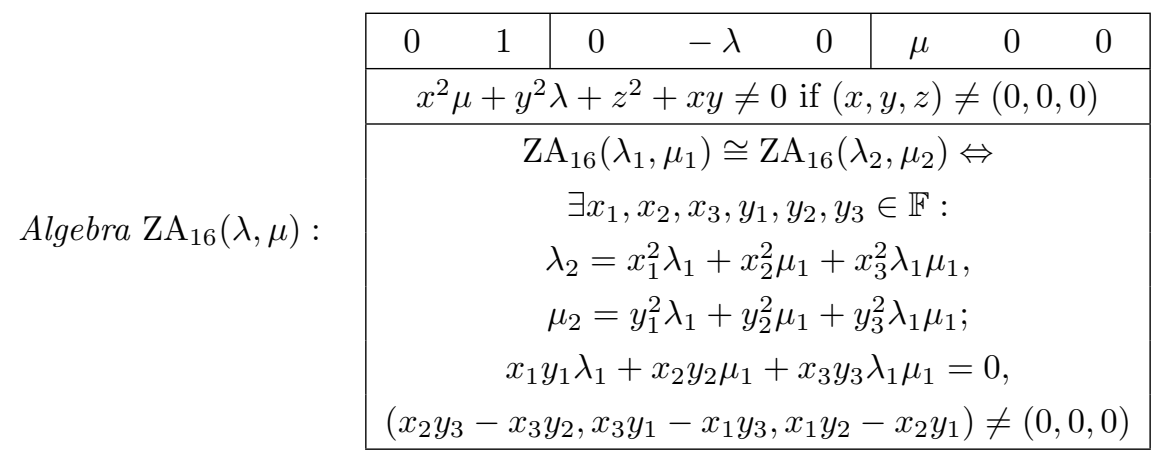

\section{Special cases}

As a consequence of our classification, we may formulate some results of independent interest.

Theorem 7.1. Each three-dimensional Lie algebra is isomorphic to one of the following algebras:

$$
\mathrm{ZA}_{1}, \mathrm{ZA}_{2}(\lambda), \mathrm{ZA}_{3}, \mathrm{ZA}_{6}, \mathrm{ZA}_{7}, \mathrm{ZA}_{8}(\lambda), \mathrm{ZA}_{9}, \mathrm{ZA}_{10}(\lambda), \mathrm{ZA}_{16}(\lambda, \mu) \text {. }
$$

Observation 7.2. The complete list of three-dimensional zeropotent algebras over an arbitrary field is as follows:

$$
\mathrm{ZA}_{1}, \mathrm{ZA}_{2}(\lambda), \mathrm{ZA}_{3}, \mathrm{ZA}_{4}, \mathrm{ZA}_{5}, \mathrm{ZA}_{6}, \mathrm{ZA}_{7}, \mathrm{ZA}_{9}, \mathrm{ZA}_{14}(\lambda) \text {. }
$$

The algebras over special fields are:

$$
\mathrm{ZA}_{8}(\lambda), \mathrm{ZA}_{10}(\lambda), \mathrm{ZA}_{11}(\lambda), \mathrm{ZA}_{12}(\lambda), \mathrm{ZA}_{13}, \mathrm{ZA}_{15}(\lambda, \mu), \mathrm{ZA}_{16}(\lambda, \mu) .
$$

There is only one algebra of the type $\mathrm{ZA}_{8}(\lambda)$ and only one of the type $\mathrm{ZA}_{12}(\lambda)$ over a field with characteristic different from two since the quotient of two nonsquare elements is a square. In characteristic 2 every element is a square, hence 
there are no algebras of the type $\mathrm{ZA}_{10}(\lambda)$. The same holds for the algebras of the type $\mathrm{ZA}_{12}(\lambda)$.

The trace of an element $\lambda \in \mathbb{F}$ is

$$
\operatorname{tr}(\lambda)=\lambda+\lambda^{2}+\ldots+\lambda^{2^{n-1}}
$$

If $x^{2}+x+\lambda=0$ for some $x \in \mathbb{F}$, then $\operatorname{tr}(\lambda)=0$, otherwise $\operatorname{tr}(\lambda)=1$. If $\mathrm{ZA}_{11}\left(\lambda_{1}\right)$ and $\mathrm{ZA}_{11}\left(\lambda_{2}\right)$ are two different algebras, then $\operatorname{tr}\left(\lambda_{1}\right)=\operatorname{tr}\left(\lambda_{2}\right)=1$ and $\operatorname{tr}\left(\lambda_{1}+\lambda_{2}\right)=0$ due to additivity of the trace. Hence, there exists an $x \in \mathbb{F}$ such that $x^{2}+x+\lambda_{1}+\lambda_{2}=0$ and the algebras $\mathrm{ZA}_{11}\left(\lambda_{1}\right)$ and $\mathrm{ZA}_{11}\left(\lambda_{2}\right)$ are isomorphic.

$\mathrm{ZA}_{13}$ is one algebra over a field with characteristic different from 2. As already said, there are no algebras in the third class $(0,1)$.

Since the number of the algebras which exist over any finite field $\mathbb{F}=\operatorname{GF}\left(p^{n}\right)$ is $2 p^{n}+7$, we can make the following conclusion.

Theorem 7.3. The number of zeropotent algebras over a finite field $\operatorname{GF}\left(p^{n}\right)$ is $2^{n+1}+8$ if $p=2$ and $2 p^{n}+10$ otherwise.

If the underlying field is algebraically closed, there are no algebras in the third class $(0,1)$ which implies:

Theorem 7.4. The complete list of three-dimensional zeropotent algebras over an algebraically closed field:

$\mathrm{ZA}_{1}, \mathrm{ZA}_{2}(\lambda), \mathrm{ZA}_{3}, \mathrm{ZA}_{4}, \mathrm{ZA}_{5}, \mathrm{ZA}_{6}, \mathrm{ZA}_{7}, \mathrm{ZA}_{9}, \mathrm{ZA}_{13}, \mathrm{ZA}_{14}(\lambda)$.

The algebra $\mathrm{ZA}_{13}$ is included only if $\operatorname{chr} \mathbb{F} \neq 2$.

As expected, this result agrees with [2, Theorem 8.1]. Our simplified notation

$$
\begin{array}{l|ll|lll|lll|}
\text { Algebra ZA : } & \sigma & \tau & \pi_{1} & \pi_{2} & \pi_{3} & \rho_{1} & \rho_{2} & \rho_{3} \\
\hline
\end{array}
$$

corresponds to the matrix

$$
A=\left[\begin{array}{ccc}
\rho_{1} & \rho_{2} & \rho_{3} \\
-\pi_{1} & -\pi_{2} & -\pi_{3} \\
\sigma & 0 & \tau
\end{array}\right]
$$

(called the structure matrix in [2]) which determines a multiplication in the algebra with the same name $A$. 
Using [2, Corollary 2.3] we find the following correspondence:

\begin{tabular}{|l|cccccc|}
\hline$A$ in $[2]$ & $A_{0}$ & $A_{1}$ & $A_{2}$ & $A_{3}$ & $A_{4}(0)$ & $A_{4}(a \neq 0)$ \\
$\mathrm{ZA}$ here & $\mathrm{ZA}_{1}$ & $\mathrm{ZA}_{3}$ & $\mathrm{ZA}_{2}(0)$ & $\mathrm{ZA}_{6}$ & $\mathrm{ZA}_{7}$ & $\mathrm{ZA}_{2}(\lambda \neq 0)$ \\
\hline$A$ in $[2]$ & $A_{5}$ & $A_{6}$ & $A_{7}(0)$ & $A_{7}(a \neq 0)$ & $A_{8}$ & $A_{9}$ \\
$\mathrm{ZA}$ here & $\mathrm{ZA}_{4}$ & $\mathrm{ZA}_{5}$ & $\mathrm{ZA}_{9}$ & $\mathrm{ZA}_{14}(\lambda \neq 3)$ & $\mathrm{ZA}_{13}$ & $\mathrm{ZA}_{14}(3)$ \\
\hline
\end{tabular}

In the case of real numbers it is obvious that the only algebras in the classes $(0,0)$ and $(0,1)$ there are the algebras $\mathrm{ZA}_{8}(-1), \mathrm{ZA}_{12}(-1)$ and $\mathrm{ZA}_{13}$.

Sylvester's criterion shows that $x^{2} \mu+y^{2} \lambda+z^{2}+x y \omega \neq 0$ for $(x, y, z) \neq(0,0,0)$ exactly when $\lambda>0, \mu>0$ and $4 \lambda \mu>\omega$.

The multiplication tables for the algebras $\mathrm{ZA}_{15}(\lambda, \mu)$ and $\mathrm{ZA}_{16}(\lambda, \mu)$ can be simplified by the following transformation:

$$
\hat{a}=\lambda^{-1 / 2} a, \hat{b}=y b, \hat{c}=y \lambda^{-1 / 2} c,
$$

where $y \neq 0$ and $\left.\left(y-\lambda^{1 / 2}\right)\right) \omega=0$. This results in $\hat{\lambda}=1$ and $\hat{\mu}=y^{2} \mu$.

If $\omega=1$, we get a one-parameter family of non-isomorphic algebras $\mathrm{ZA}_{15}(1, \mu>$ $\left.\frac{1}{4}\right)$. In the case $\omega=0$, we can choose $y=\mu^{-1 / 2}$ in order to get $\hat{\mu}=1$ and thus obtain the usual algebra of geometric three-dimensional vectors equipped with the cross product, i.e., $A_{16}(1,1)$.

Theorem 7.5. A three-dimensional zeropotent algebra over the field of real numbers is isomorphic to one of the following algebras:

$$
\begin{gathered}
\mathrm{ZA}_{1}, \mathrm{ZA}_{2}(\lambda), \mathrm{ZA}_{3}, \mathrm{ZA}_{4}, \mathrm{ZA}_{5}, \mathrm{ZA}_{6}, \mathrm{ZA}_{7}, \mathrm{ZA}_{8}(-1), \mathrm{ZA}_{9}, \\
\mathrm{ZA}_{12}(-1), \mathrm{ZA}_{13}, \mathrm{ZA}_{14}(\lambda), \mathrm{ZA}_{15}\left(1, \mu>\frac{1}{4}\right), \mathrm{ZA}_{16}(1,1) .
\end{gathered}
$$

The result agrees with [4, Theorem 6.1]. The correspondence is as follows:

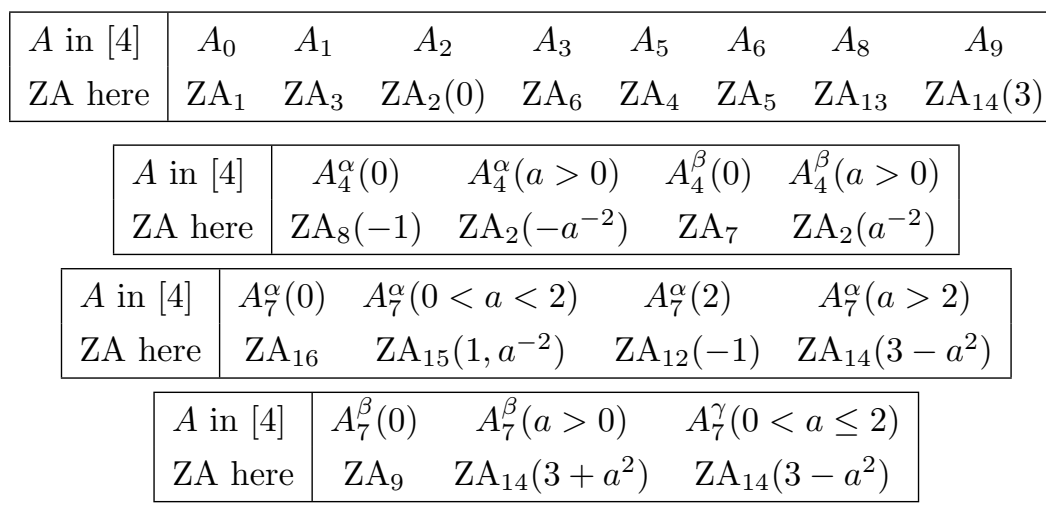

Acknowledgement. The authors would like to thank the referee for valuable suggestions and comments. 


\title{
References
}

[1] A. Fowler-Wright, The Classification of Three-Dimensional Lie Algebras, Ph.D. Thesis, The University of Warwick, Coventry, 2014.

[2] Y. Kobayashi, K. Shirayanagi, S. Takahasi and M. Tsukada, Classification of three-dimensional zeropotent algebras over an algebraically closed field, Comm. Algebra, 45(12) (2017), 5037-5052.

[3] E. N. Kuzmin, Binary Lie algebras of small dimension, Algebra and Logic, 37(3) (1998), 181-186.

[4] K. Shirayanagi, S. Takahasi, M. Tsukada and Y. Kobayashi, Classification of three-dimensional zeropotent algebras over the real number field, Comm. Algebra, 46(11) (2018), 4663-4681.

\author{
Anton Cedilnik \\ Biotechnical Faculty \\ University of Ljubljana \\ SI 1000 Ljubljana, Slovenia \\ e-mail: anton.cedilnik@gmail.com \\ Marjan Jerman (Corresponding Author) \\ Faculty of Mathematics and Physics \\ University of Ljubljana \\ SI 1000 Ljubljana, Slovenia \\ e-mail: marjan.jerman@fmf.uni-lj.si
}

